\title{
Analysis of a virus-resistant HIV-1 model with behavior change in non-progressors
}

\author{
Rabiu Musa, Robert Willie, Nabendra Parumasur \\ School of Mathematics, Statistics and Computer Science \\ University of KwaZulu-Natal, Durban, South Africa. \\ rabiumusa003@gmail.com, willier@ukzn.ac.za, parumasurn1@ukzn.ac.za
}

Received: 26 February 2020, accepted: 14 June 2020, published: 8 August 2020

\begin{abstract}
We develop a virus-resistant HIV-1 mathematical model with behavior change in HIV1 resistant non-progressors which was analyzed for both partial and total abstinence cases. The model has both disease-free and endemic equilibrium points that are locally asymptotically stable depending on the value of the associated threshold quantities $\mathcal{R}_{T}$ and $\mathcal{R}_{T}^{\prime}$. In both cases, a nonlinear Goh-Volterra Lyapunov function was used to prove that the endemic equilibrium point is globally asymptotically stable for special case while the method of Castillo-Chavez was used to prove the global asymptotic stability of the disease-free equilibrium point. In both the analytic and numerical results, this study shows that in the context of resistance to HIV/AIDS, total abstinence can also play an important role in protection against this notorious infectious disease.
\end{abstract}

Keywords-Resistance; Behavior change; Partial \& Total Abstinence; Goh-Volterra Lyapunov function.

AMS Subject Classification: 92Bxx, 92B05.

\section{INTRODUCTION}

As it was reported in the 1980s, the human immunodeficiency virus (HIV), and the later stage of infection through cell depletion known as AIDS has continue to play a leading role in the series of the greatest ever infectious disease. United Nations Program on HIV/AIDS (UNAIDS) and the World Health Organization (WHO) have already provided the estimates of the number of cases since the 1980s.

More than 30 million people are currently HIV positive. According to the current trends, at least 7300 people are infected with HIV and a minimum of 5000 die from AIDS-related causes including at-least 690 children on a daily basis (UNAIDS, 2009). This means that for every five HIV positive individuals, at least four of them including adults and children die from the infection daily [10], [32]. The two main types of HIV are HIV-1 and HIV2. The most dangerous that has spread worldwide is HIV-1 while the latter is less pathogenic and less spread since it's confined to West African countries. The test carried out on one can not

\footnotetext{
Copyright: (c) 2020 Musa et al. This article is distributed under the terms of the Creative Commons Attribution License (CC BY 4.0), which permits unrestricted use, distribution, and reproduction in any medium, provided the original author and source are credited.
}

Citation: Rabiu Musa, Robert Willie, Nabendra Parumasur, Analysis of a virus-resistant HIV-1 model

with behavior change in non-progressors, Biomath 9 (2020), 2006143,

http://dx.doi.org/10.11145/j.biomath.2020.06.143

Page 1 of 17 
sufficiently detect the other due to large genetic differences between them.

Immediately after HIV infection, the lymphocytes, or white blood cells, known as CD4+ T cells are the major target. Therefore, the antiHIV antibody and cytotoxic $\mathrm{T}$ cell production by the immune system is consequently initiated. An HIV positive individual is not classified as having AIDS until CD4+T cell count which is approximately around $1000 \mathrm{~mm}^{-3}$ depletes to $150 \mathrm{~mm}^{-3}$ or thereabout. Since CD4+T plays a very important role in the body immune mechanism, deterioration and depletion result in acquired immunodeficiency syndrome called AIDS. The average number of times it takes HIV to develop to AIDS is dependent on the strength of the immune system of the victim [23].

It is therefore pertinent to study methods of HIV prevention. Different control strategies such as behavior change due to HIV awareness campaign, reduction in sexual partners, anti retroviral treatment ART etc. have collectively played important roles in combating the menace. They are still very much relevant due to unavailability of vaccine. The use of condom has also played an important role and can possibly prevent HIV transmission almost perfectly. Other intervention methods that can concurrently prevent both sexes are still very much needed. Recently, an experimental product containing a drug that can prevent rectal and vaginal transmission of HIV and other sexually transmitted diseases was detected but unfortunately did not see the light of the day due to the fact that the gel is ineffective with high HIV infection risk [24]. Other efforts such as the HIV vaccine and diaphragm technique fail to manifest to any meaningful impact [6], [21].

From biological point of view, HIV resistance is known as the genetic mutation in the DNA that delays AIDS progression or aids production of permanent immunity (i.e. no progression) to AIDS. This kind of mutation which is known as CCR5-delta 32 plays an important role in the development of the two kinds of HIV resistance known. This CCR5-delta 32 breaks and distorts the HIV's ability to deplete and destroy the immunity of the CD4+T cells. The mutation makes the CCR5 co-receptor on the outside of cells to develop at a smaller rate than usual and no longer sit outside of the cell. This co-receptor is similar to a door that allows HIV passage into the cell where within a second locks "the door" which consequently prevents HIV entrance into the CD4+T cells [14]. This genetic mutation has been reported to be inborn. There are very few paper on resistant mathematical model, some of them are [25], [13] and [15] but the resistance was modeled on influenza and SARS which is quite different from HIV/AIDS. This still remains a biological research question needed to be answered.

Research has shown that some people develop resistance to the killer HIV-1 virus [22], [28]. In fact, a report in [18] shows that though this resistance is rare but actually exists. Virus resistance can be understood in two scenarios. First, there are cases of individuals that are exposed to HIV but after a long period of times, diagnosis shows that they are uninfected. This case of exposed uninfected have been detected from among infants of infected mothers, health workers during treatment of infected individuals, commercial sex workers, individuals having unprotected sex with seropositive partners etc. The second category is HIV infected individuals with low or no progression to AIDS as expected under normal circumstances. They live with the virus for many years with an absolutely low level of HIV-1 RNA or no loss of CD4+ cells that has been identified among various individuals such as children and homosexual men and women mutation [18], [8].

In 2014, the report in [12] confirms that some people show partial or absolutely complete inborn resistance to the HIV virus. The major or main contributor to this strange development is a mutation of the gene encoding CCR5 which acts as a co-receptor for HIV. CCR5 may even be defective in some individuals which will enhance protection against disease. These individuals live a normal life since the HIV-1 virus cannot bind itself to it and its perhaps here that the key to 
overcome the disease lies hidden. Estimation later shows that the proportion of individuals under this category is less than $1 \%$. Similar occurrences make leading Oxford University researcher Sarah Rowland-Jones to believe continual exposure is a requirement for maintaining immunity after which 15 proteins were identified to be unique to those virus-free sex workers [3], [2]. A genetic mutation that blocks HIV which may hold the key to future treatment was also studied in 2016 by [9].

In 2010, [4] identified factors such as APOBEC3G, Toll-like receptors, acute-phase amyloid A protein, interleukin-22, APOBEC3G and natural killer cells as the main reason why some people do not even seroconvert let alone progressing to AIDS despite multiple HIV exposure. More interesting reasons behind this strange occurrence has been examined by the university of Minnesota in 2014 [33] and by [17] in 2013. Another interesting factor that influence the spread of HIV/AIDS is change in sexual behavior towards sex. This is caused by the infectiousness nature, high death rate and stigmatization encountered by victims of HIV/AIDS. This has subsequently affect the transmission of the disease in recent years.

Behavior change intervention will help individuals change their drug-using behaviors and sexual behavior that put them at a high risk of contracting HIV. It also creates skills and knowledge that can influence their motivation and ability to kick start behavior change. Couples, peer groups, individuals, communities or institutions can be targeted on a multiple level. This behavior change can also be motivated through skills-building, motivational or educational approach. Interventions can target different kind of behaviors such as condom usage, number of sexual partners, correct use of best prevention approach etc. Though many researchers have developed different models to examine the dynamics of the virus, HIV-1 mathematical model where infected individuals gain resistance to acquisition of HIV and resistance to deterioration of HIV incorporating behavior change in form of partial and total abstinence is still a biological question needed to be answered.
Researchers like [31], [19] have done commendable work in tackling the menace of the deadly virus, in this research, we present a new virusresistant HIV-1 model with behavior change. This behavior change to avoid infection happens as a result of the wide spread of the agony and death caused by HIV/AIDS. This change happens either partially or totally. Those who show partial abstinence are those that only reduced their sexual partners but still involve in HIV-risk activities or live in endemic environment while those who totally abstain are those who maintain only one sexual partner and do away from all HIV-risk activities or exposed and endemic environment.

Mathematical modeling has become an effective tool in studying infectious disease by many researchers. It shall be used again here to study the dynamics of resistance in HIV-1 transmission and how it produce significant reduction rate in the community. We hope it helps policy-makers and public health workers in the epidemic control.

Several researchers like [20], [19], [1] and references therein have published commendable research output about transmission dynamics of HIV/AIDS. They have also studied control and prevention strategies of this notorious epidemic. In order to further extend, compliment and contribute to the work of the aforementioned researchers, a new comprehensive model has been designed. The model extends the work of the aforementioned researchers by, for instance,

1) Considering the influence of virus-resistance i.e. resistance to acquisition and resistance to deterioration.

2) Incorporating the change of behavior class whose rate of progression is either through partial abstinence or total abstinence.

3) Including a compartment $\left(I_{1}\right)$ for slow progressors. These are the category of people with partial resistance to the virus.

4) Including a compartment $\left(I_{2}\right)$ for non progressors. These are the category of people with complete resistance to the virus and do not move to AIDS compartment $(A)$.

5) Including a compartment $\left(I_{3}\right)$ for fast pro- 
R. Musa, R. Willie, N. Parumasur, Analysis of a virus-resistant HIV-1 model with behavior change in ...

gressors. These are the category of people with no resistance to the virus.

All these instances have not been considered before.

The paper is organized as follows. Section 2 entails model formulation and assumptions while section 3 contains basic properties of the model. This is followed by the analysis of the sub-model (model with total abstinence) and that of the full model (model with partial abstinence) in section 4. Section 5 presents the numerical simulation and discussion of results while the last section contains the conclusion, acknowledgment and disclosure statement.

\section{Model Formulation And Model ASSUMPTIONS}

We formulate an HIV-1 resistant and behavior change model by splitting the total human population at time $t$, denoted by $N(t)$, into six mutuallyexclusive compartments of susceptible individuals $S(t)$, slow progressor HIV-1 infected class $I_{1}(t)$, non progressor HIV-1 infected class $I_{2}(t)$, fast progressor HIV-1 infected class $I_{3}(t)$, behavior change class $I_{4}$ and AIDS class $A$ such that

$N(t)=S(t)+I_{1}(t)+I_{2}(t)+I_{3}(t)+I_{4}(t)+A(t)$.

It is worth noting that the AIDS class consists of weak and unhealthy infected individuals that are assumed to be sexually inactive.

Sexually active individuals are recruited into the susceptible population at a constant rate $B$. The susceptible individuals acquire the virus through effective contact with an HIV-1 positive and infectious individuals at the rate $\lambda$ given by

$$
\lambda=\frac{\beta\left(I_{3}+\sigma_{1} I_{1}+\sigma_{2} I_{2}+\sigma_{3} I_{4}\right)}{N},
$$

where $\beta$ in (1) denotes the effective contact rate that is capable of leading to infection, $0 \leq \sigma_{1} \leq 1$ denotes the modification parameter that account for the assumed reduction in the transmission of virus by the slow progressor HIV-1 infected class $I_{1}$ in comparison to the fast progressor HIV-1 infected individuals in $I_{3}, 0 \leq \sigma_{2}, \sigma_{3} \leq 1$ are the modification parameters accounting for the assumed reduction of infectiousness by $I_{2}$ and $I_{4}$ classes in comparison to the slow-progressor and fast progressor classes $I_{1}$ and $I_{3}$ respectively. So that

$$
\sigma_{3}<\sigma_{2}<\sigma_{1}<1, \quad \sigma_{3} \geq 0 .
$$

The acquisition of infection by the slow progressor HIV-1 infected individuals $I_{1}$ occur at the rate $\alpha_{1} \lambda$, that of $I_{2}$ occur at the rate $\alpha_{2} \lambda$ and that of $I_{3}$ at the rate $\alpha_{3} \lambda$. Natural death occur constantly to anybody at the rate $\mu$ and rate of progression from $I_{1}$ to AIDS class $A$ at the rate $\rho_{1}$. Therefore, the rate of change of the total population of the susceptible and and slow progressor classes is respectively given by

$$
\begin{aligned}
\dot{S}(t) & =B-\left(\alpha_{1}+\alpha_{2}+\alpha_{3}\right) \lambda S-\mu S, \\
\dot{I}_{1}(t) & =\alpha_{1} \lambda S-\rho_{1} I_{1}-\mu I_{1},
\end{aligned}
$$

where represents derivative with respect to time. The non-progressor HIV-1 infected class is generated by the break-through of infection of susceptible class at the rate $\alpha_{2} \lambda$, total abstinence due to behavior change at the rate $\gamma_{1}$, partial abstinence from $I_{4}$ due to behavior change at the rate $\gamma_{2}$ and natural death at the rate $\mu$ so that we have

$$
\dot{I}_{2}(t)=\alpha_{2} \lambda S-\gamma_{1} I_{2}+\gamma_{2} I_{4}-\mu I_{2} .
$$

Similarly, we compose the fast progressor class by the break-through of infection of the susceptible class at the rate $\alpha_{3} \lambda$, AIDS acquisition at the rate $\rho_{2}$ so that the class is given by

$$
\dot{I}_{3}(t)=\alpha_{3} \lambda S-\rho_{2} I_{3}-\mu I_{3} .
$$

The behavior change class is formulated through the total abstinence of non progressors at the rate $\gamma_{1}$ and partial abstinence at the rate $\gamma_{2}$ given by

$$
\dot{I}_{4}(t)=\gamma_{1} I_{2}-\gamma_{2} I_{4}-\mu I_{4} \text {. }
$$

While incorporating the behavior change in the model, we deliberately focused on the behavior change of the non-progressors HIV-1 infected individuals even though, it is imperative that all individuals can change their behavior at any given time. This is because this class of individuals are the most dangerous class just that they won't show 
R. Musa, R. Willie, N. Parumasur, Analysis of a virus-resistant HIV-1 model with behavior change in ...

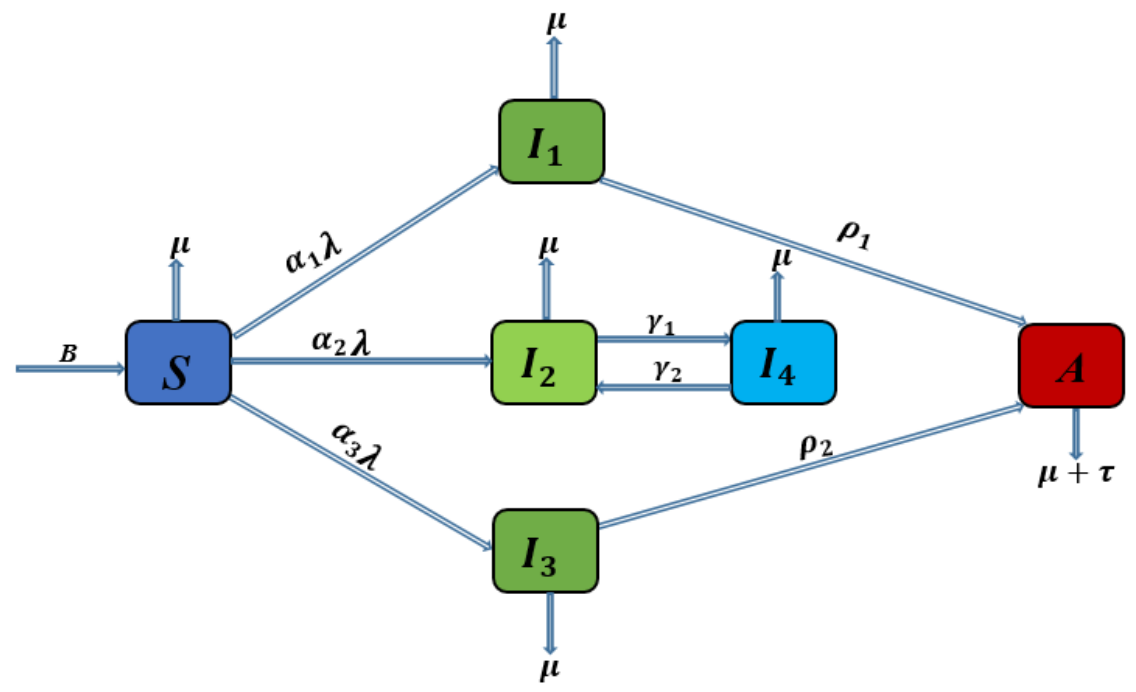

Fig. 1. Flow chart of the model.

any sign of AIDS.

And finally, the AIDS class is given by

$$
\dot{A}(t)=\rho_{1} I_{1}+\rho_{2} I_{3}-(\mu+\tau) A,
$$

where $\tau$ is the AIDS-induced death rate. Since progression are not the same, we have

$$
\alpha_{3}>\alpha_{1}>\alpha_{2}, \alpha_{1}+\alpha_{2}+\alpha_{3}=1
$$

where $0<\alpha_{1}, \alpha_{2}, \alpha_{3}<1$. The resultant mathematical model for the transmission dynamics of HIV-1 incorporating virus resistance and behavior change through partial and total abstinence using a set of non-linear autonomous set of differential equations is given by:

$$
\begin{aligned}
\frac{d S}{d t} & =B-\left(\alpha_{1}+\alpha_{2}+\alpha_{3}\right) \lambda S-\mu S, \\
\frac{d I_{1}}{d t} & =\alpha_{1} \lambda S-K_{1} I_{1}, \\
\frac{d I_{2}}{d t} & =\alpha_{2} \lambda S+\gamma_{2} I_{4}-K_{2} I_{2}, \\
\frac{d I_{3}}{d t} & =\alpha_{3} \lambda S-K_{3} I_{3}, \\
\frac{d I_{4}}{d t} & =\gamma_{1} I_{2}-K_{4} I_{4}, \\
\frac{d A}{d t} & =\rho_{1} I_{1}+\rho_{2} I_{3}-K_{5} A,
\end{aligned}
$$

where

$$
\begin{aligned}
& K_{1}=\rho_{1}+\mu, K_{2}=\gamma_{1}+\mu, K_{3}=\rho_{2}+\mu, \\
& K_{4}=\gamma_{2}+\mu, K_{5}=\mu+\tau,
\end{aligned}
$$

with initial condition

$$
\begin{aligned}
& S(0)>0, I_{1}(0)>0, I_{2}(0)>0, \\
& I_{3}(0)>0, I_{4}(0)>0, A(0)>0 .
\end{aligned}
$$

The flow chart of this model is given in Figure 1.

III. BASIC Properties OF THE MODEL

Since the model is a dynamical system, it it is therefore imperative to ensure that it is biologically meaningful through the establishment of its positivity solution and boundedness at all time $t \geq 0$.

A. Positivity and boundedness of the Model.

Lemma III.1. The closed set

$\Gamma=\left\{\left(S, I_{1}, I_{2}, I_{3}, I_{4}, A\right) \in \mathbb{R}_{+}^{6} \mid S+I_{1}+\ldots+I_{4}+A \leq \frac{B}{\mu}\right\}$

is attracting and positively invariant with respect to the model equation (4)-(9).

Proof: From (4), we define an integrating factor as $\xi(t)=\exp \left\{\int_{o}^{t}\left[\mu+\left(\alpha_{1}+\alpha_{2}+\alpha_{3}\right) \lambda(\eta)\right] d \eta\right\}$, 
R. Musa, R. Willie, N. Parumasur, Analysis of a virus-resistant HIV-1 model with behavior change in ...

where $\lambda(\eta)=\lambda\left(I_{1}, I_{2}, I_{3}, I_{4}\right)$. So that the solution of (4) is given by

$$
S(t) \xi(t)=B \int_{o}^{t} \xi(t) d t,
$$

which can be re-written as

$$
\begin{aligned}
& S(t) \exp \left\{\int_{o}^{t}\left[\mu+\left(\alpha_{1}+\alpha_{2}+\alpha_{3}\right) \lambda(\eta)\right] d \eta\right\}=S(0) \\
& +B \int_{o}^{t}\left[\exp \left\{\int_{o}^{s}\left[\mu+\left(\alpha_{1}+\alpha_{2}+\alpha_{3}\right) \lambda(\eta)\right] d \eta\right\}\right] d s,
\end{aligned}
$$

which implies

$$
\begin{aligned}
& S(t) \exp \left\{\mu t+\int_{o}^{t}\left(\alpha_{1}+\alpha_{2}+\alpha_{3}\right) \lambda(\eta) d \eta\right\}=S(0) \\
& +B \int_{o}^{t}\left[\exp \left\{\mu s+\int_{o}^{s}\left(\alpha_{1}+\alpha_{2}+\alpha_{3}\right) \lambda(\eta) d \eta\right\}\right] d s
\end{aligned}
$$

so that

$$
\begin{aligned}
S(t) & =B \int_{o}^{t}\left[\exp \left\{\mu s+\int_{o}^{s}\left(\alpha_{1}+\alpha_{2}+\alpha_{3}\right) \lambda(\eta) d \eta\right\}\right] d s \\
& \times \exp \left\{-\mu t-\int_{o}^{t}\left(\alpha_{1}+\alpha_{2}+\alpha_{3}\right) \lambda(\eta) d \eta\right\} \\
& +S(0) \exp \left\{-\mu t-\int_{o}^{t}\left(\alpha_{1}+\alpha_{2}+\alpha_{3}\right) \lambda(\eta) d \eta\right\},
\end{aligned}
$$

where $S(0)$ is an initial condition for $S(t)$ and hence it is a constant. This expression guarantees the positivity of the state variable $S(t)$ under the condition that $S(0)>0$ which consequently ensures the positivity of $I_{1}(t), I_{2}(t), I_{3}(t), I_{4}(t)$ and $A(t)$ provided that 10$]$ is satisfied for all time $t \geq 0$.

Furthermore, addition of (4)-(9) gives

$$
\begin{gathered}
\frac{d N(t)}{d t}=B-\mu N(t)-\tau A \\
\Downarrow \\
\frac{d N(t)}{d t} \leq B-\mu N(t),
\end{gathered}
$$

whose solution is

$$
N(t) \leq \frac{B}{\mu}+\left[N(0)-\frac{B}{\mu}\right] \exp (-\mu t),
$$

$$
\begin{aligned}
\lim _{t \rightarrow \infty} N(t) & \leq \frac{B}{\mu}+\lim _{t \rightarrow \infty}\left[N(0)-\frac{B}{\mu}\right] \exp (-\mu t) \\
& =\frac{B}{\mu} .
\end{aligned}
$$

This shows the boundedness of the solution above by $\frac{B}{\mu}$ in the domain defined by the provision of Lemma III.1. Therefore, the model is epidemically well-posed and mathematically meaningful since all the state variables are non-negative for all $t \geq$ 0 . Hence, it is sufficient to study and analyze the model in $\Gamma$ [26], [27]. This completes the proof.

\section{AnAlysis of THE Model}

A. Analysis of the Model with Total Abstinence of Non-progressors

Here, we analyze the model for non-progressors that change their behavior through total abstinence from all means of contracting HIV-1 and from all HIV-1 endemic environments i.e. $\gamma_{2}=0, \sigma_{3}=0$ so that equation (4)-(9) becomes

$$
\begin{aligned}
\frac{d S}{d t} & =B-\left(\alpha_{1}+\alpha_{2}+\alpha_{3}\right) \lambda_{1} S-\mu S, \\
\frac{d I_{1}}{d t} & =\alpha_{1} \lambda_{1} S-K_{1} I_{1}, \\
\frac{d I_{2}}{d t} & =\alpha_{2} \lambda_{1} S-K_{2} I_{2}, \\
\frac{d I_{3}}{d t} & =\alpha_{3} \lambda_{1} S-K_{3} I_{3}, \\
\frac{d I_{4}}{d t} & =\gamma_{1} I_{2}-\mu I_{4}, \\
\frac{d A}{d t} & =\rho_{1} I_{1}+\rho_{2} I_{3}-K_{5} A,
\end{aligned}
$$

where

$$
\lambda_{1}=\frac{\beta\left(I_{3}+\sigma_{1} I_{1}+\sigma_{2} I_{2}\right)}{N} .
$$

All model parameters are positive.

\section{B. Local Stability of Disease-Free equilibrium (DFE)}

The disease-free equilibrium of (13)-(18) is given by

$$
\begin{aligned}
\psi_{1}^{*} & =\left(S^{*}, I_{1}^{*}, I_{2}^{*}, I_{3}^{*}, I_{4}^{*}, A^{*}\right) \\
& =\left(\frac{B}{\mu}, 0,0,0,0,0\right) .
\end{aligned}
$$

This shows that

$$
N^{*}=S^{*}=\frac{B}{\mu} \text { and } \frac{S^{*}}{N^{*}}=1
$$


R. Musa, R. Willie, N. Parumasur, Analysis of a virus-resistant HIV-1 model with behavior change in ...

at disease-free equilibrium point $\psi_{1}^{*}$. By employing the next generation method [7], [34], $\mathcal{F}_{1}$ (the new infection terms) and $\mathcal{V}_{1}$ (transfer terms) are expressed as

$$
\begin{gathered}
\mathcal{F}_{1}=\left[\begin{array}{ccccc}
\alpha_{1} \sigma_{1} \beta & \beta \sigma_{2} \alpha_{1} & \beta \alpha_{1} & 0 & 0 \\
\alpha_{2} \sigma_{1} \beta & \beta \sigma_{2} \alpha_{2} & \beta \alpha_{2} & 0 & 0 \\
\alpha_{3} \sigma_{1} \beta & \beta \sigma_{2} \alpha_{3} & \beta \alpha_{3} & 0 & 0 \\
0 & 0 & 0 & 0 & 0 \\
0 & 0 & 0 & 0 & 0
\end{array}\right], \\
\mathcal{V}_{1}=\left[\begin{array}{ccccc}
K_{1} & 0 & 0 & 0 & 0 \\
0 & K_{2} & 0 & 0 & 0 \\
0 & 0 & K_{3} & 0 & 0 \\
0 & -\gamma_{1} & 0 & \mu & 0 \\
-\rho_{1} & 0 & -\rho_{2} & 0 & K_{5}
\end{array}\right]
\end{gathered}
$$

Taking $\rho$ as the spectral radius (magnitude of the dominate eigenvalue) of the next generation matrix $\mathcal{F}_{1} \mathcal{V}_{1}^{-1}$, the reproduction number is given by

$$
\mathcal{R}_{T}^{\prime}=\frac{\beta\left(\alpha_{1} K_{2} K_{3} \sigma_{1}+\alpha_{2} K_{1} K_{3} \sigma_{2}+\alpha_{3} K_{1} K_{2}\right)}{K_{1} K_{2} K_{3}} .
$$

The quantity $\mathcal{R}_{T}^{\prime}$ represents the measure of average number of new virus infection of HIV-1 developed by a single HIV-1 infected individual in a population where there are people who practice total abstinence and are completely susceptible. Hence, we present the following Lemma.

Lemma IV.1. The DFE of the reduced model (13)(18) with total abstinence is locally asymptotically stable (LAS) if $\mathcal{R}_{T}^{\prime}<1$, and unstable if $\mathcal{R}_{T}^{\prime}>1$.

The proof is standard and can be established using theorem 2 of [34].

\section{Existence of Endemic Equilibrium}

The reduced model with total abstinence has a unique positive endemic equilibrium point (EEP). This is the point where at least one of the virus infected compartments is non-zero. Let

$$
\psi_{1}^{* *}=\left(S^{* *}, I_{1}^{* *}, I_{2}^{* *}, I_{3}^{* *}, I_{4}^{* *}, A^{* *}\right)
$$

be the endemic equilibrium point. We further define the force of infection as

$$
\lambda_{1}^{* *}=\frac{\beta\left(I_{3}^{* *}+\sigma_{1} I_{1}^{* *}+\sigma_{2} I_{2}^{* *}\right)}{N^{* *}} .
$$

Solving equation (13)-(18) in terms of the force of infection $\lambda_{1}^{* *}$ at steady-state gives:

$$
\begin{aligned}
S^{* *} & =\frac{B}{\mu+\left(\alpha_{1}+\alpha_{2}+\alpha_{3}\right) \lambda_{1}^{* *}}, \\
I_{1}^{* *} & =\frac{\alpha_{1} B \lambda_{1}^{* *}}{K_{1}\left[\mu+\left(\alpha_{1}+\alpha_{2}+\alpha_{3}\right) \lambda_{1}^{* *}\right]}, \\
I_{3}^{* *} & =\frac{B \lambda_{1}^{* *}}{K_{3}\left[\mu+\left(\alpha_{1}+\alpha_{2}+\alpha_{3}\right) \lambda_{1}^{* *}\right]}, \\
I_{4}^{* *} & =\frac{\gamma_{1} B \alpha_{2} \lambda_{1}^{* *}}{K_{2} \mu\left[\mu+\left(\alpha_{1}+\alpha_{2}+\alpha_{3}\right) \lambda_{1}^{* *}\right]}, \\
A^{* *} & =\frac{B \lambda_{1}^{* *}\left(\rho_{1} \alpha_{1} K_{3}+K_{1} \rho_{2}\right)}{K_{1} K_{3} K_{5}\left[\mu+\left(\alpha_{1}+\alpha_{2}+\alpha_{3}\right) \lambda_{1}^{* *}\right]}, \\
I_{2}^{* *} & =\frac{\alpha_{2} B \lambda_{1}^{* *}}{K_{2}\left[\mu+\left(\alpha_{1}+\alpha_{2}+\alpha_{3}\right) \lambda_{1}^{* *}\right]}, \\
N^{* *} & =\frac{B K_{1} K_{3} K_{5} f_{1}-\tau B \lambda_{1}^{* *}\left(\rho_{1} \alpha_{1} K_{3}+K_{1} \rho_{2}\right)}{\mu K_{1} K_{3} K_{5} f_{1}},
\end{aligned}
$$

where $f_{1}=\mu+\left(\alpha_{1}+\alpha_{2}+\alpha_{3}\right) \lambda_{1}^{* *}$. Substituting all the equations in (24) into (23), it can be shown that the non-zero equilibria of the model satisfy the following linear equation in terms of $\lambda_{1}^{* *}$ :

$$
a_{o} \lambda_{1}^{* *}+a_{1}=0
$$

where

$$
\begin{aligned}
a_{o} & =\alpha_{1} \mu K_{2} K_{3}\left(\mu+\tau+\rho_{1}\right) \\
& +K_{1} K_{2}\left[\mu \alpha_{3}\left(\rho_{2}+\mu+\tau\right)+K_{3} K_{5} \alpha_{2}\right], \\
a_{1} & =\mu K_{1} K_{2} K_{3} K_{5}\left(1-\mathcal{R}_{T}^{\prime}\right) .
\end{aligned}
$$

Clearly, $a_{o}>0, a_{1} \geq 0$ if and only if $\mathcal{R}_{T}^{\prime} \leq 1$ so that $\lambda_{1}^{* *}=-\frac{a_{1}}{a_{o}} \leq 0$. This shows that no existence of positive endemic equilibrium whenever $\mathcal{R}_{T}^{\prime} \leq 1$. Hence, the endemic equilibrium point $\psi_{1}^{* *}$ exists and unique whenever $\mathcal{R}_{T}^{\prime}>1$. We claim the following result.

Lemma IV.2. The endemic equilibrium point (EEP) of the reduced model (13)-(18) with total abstinence is locally asymptotically stable (LAS) if $\mathcal{R}_{T}^{\prime}>1$.

\section{Global Stability of DFE}

To establish the global stability of DFE points, we adopt the approach of [5] to re express (13)(18) in the following vector form

$$
\dot{X}=L(X, Y)
$$


R. Musa, R. Willie, N. Parumasur, Analysis of a virus-resistant HIV-1 model with behavior change in ...

and

$$
\dot{Y}=M(X, Y), M(X, 0)=0
$$

where the vector $X=(S)$ denotes the HIV-1 uninfected compartment of the system and $Y=\left(I_{1}, I_{2}, I_{3}, I_{4}, A\right) \in \mathbb{R}_{+}^{5}$ represents the HIV1 infected compartments. Using the DFE point to establish the stability analysis, the following two conditions must be satisfied:

$\mathbf{N}_{1}$ : For $\dot{X}(t)=L\left(X^{o}, 0\right), X^{o}$ is globally asymptotically stable.

$\mathbf{N}_{2}: M(X, Y)=J Y-\hat{M}(X, Y), \hat{M}(X, Y) \geq 0$ for $X, Y \in \Omega_{m}$ where $J=\frac{\partial M}{\partial Y}\left(X^{o}, 0\right)$.

For this analysis, the expressions for $J_{1}, Y_{1}, \hat{M}_{1}$ and $M_{1}$ are for the reduced model with the same definition as above while expressions for $J, Y, \hat{M}$ and $M$ are for the full model with the same definition. From our model equation, we obtain the Jacobian matrix of only the infected compartment at DFE as follows:

$$
\begin{aligned}
& J_{1}= \\
& {\left[\begin{array}{ccccc}
\frac{\alpha_{1} \sigma_{1} \beta S^{*}}{N^{*}}-K_{1} & \frac{\beta \sigma_{2} \alpha_{1} S^{*}}{N^{*}} & \frac{\beta \alpha_{1} S^{*}}{N^{*}} & 0 & 0 \\
\frac{\alpha_{2} \sigma_{1} \beta S^{*}}{N^{*}} & \frac{\alpha_{2} \sigma_{2} \beta S^{*}}{N^{*}}-K_{2} & \frac{\beta \alpha_{2} S^{*}}{N^{*}} & 0 & 0 \\
\frac{\alpha_{3} \sigma_{1} \beta S^{*}}{N^{*}} & \frac{\alpha_{3} \sigma_{2} \beta S^{*}}{N^{*}} & \frac{\beta \alpha_{3} S^{*}}{N^{*}}-K_{3} & 0 & 0 \\
0 & \gamma_{1} & 0 & -\mu & 0 \\
\rho_{1} & \rho_{2} & 0 & -K_{5}
\end{array}\right]} \\
& J_{1} Y_{1}=J_{1}\left[\begin{array}{c}
I_{1} \\
I_{2} \\
I_{3} \\
I_{4} \\
A
\end{array}\right]=\left[\begin{array}{c}
\frac{\beta \alpha_{1}\left(\sigma_{1} I_{1}+\sigma_{2} I_{2}+I_{3}\right) S^{*}}{N^{*}}-K_{1} I_{1} \\
\frac{\beta \alpha_{2}\left(\sigma_{1} I_{1}+\sigma_{2} I_{2}+I_{3}\right) S^{*}}{N^{*}}-K_{2} I_{2} \\
\frac{\beta \alpha_{3}\left(\sigma_{1} I_{1}+\sigma_{2} I_{2}+I_{3}\right) S^{*}}{N^{*}}-K_{3} I_{3} \\
\gamma_{1} I_{2}-\mu I_{4} \\
\rho_{1} I_{1}+\rho_{2} I_{3}-K_{5} A
\end{array}\right]
\end{aligned}
$$$$
\hat{M}_{1}(X, Y)=\left[\begin{array}{c}
\beta \alpha_{1}\left(\sigma_{1} I_{1}+\sigma_{2} I_{2}+I_{3}\right)\left(1-\frac{S}{N}\right) \\
\beta \alpha_{2}\left(\sigma_{1} I_{1}+\sigma_{2} I_{2}+I_{3}\right)\left(1-\frac{S}{N}\right) \\
\beta \alpha_{3}\left(\sigma_{1} I_{1}+\sigma_{2} I_{2}+I_{3}\right)\left(1-\frac{S}{N}\right) \\
0 \\
0
\end{array}\right],
$$

Since $S \leq N$, this shows that $\hat{M}_{1}(X, Y) \geq 0$. It can be seen that $\lim _{t \rightarrow \infty} X(t)=X^{o}$ and $J$ is an M-matrix, thus $X^{o}$ is globally asymptotically stable, hence, $\mathbf{N}_{1}$ is satisfied. Also, $\hat{M}_{1}(X, Y) \geq 0$ for $(X, Y) \in \Omega_{m}$. Hence, $\mathbf{N}_{2}$ is satisfied and $E^{o}$ is globally asymptotically stable whenever $\mathcal{R}_{T}^{\prime}<1$.

\section{E. Global Stability of Endemic Equilibrium Point}

Following the provision of Lemma IV.2, we establish the following theorem.

Theorem IV.3. The endemic equilibrium point of the reduced model (13)-(18) is globally asymptotically stable $(G A S)$ whenever $\mathcal{R}_{T}^{\prime}>1$.

Proof: Using the idea of [1], we construct the Lyapunov function:

$$
B=\sum_{k=1}^{6} A_{k} B_{k}, \quad A_{k}>0,
$$

where $A_{k}$ is a constant and $B_{k}$ is given by

$$
\begin{gathered}
B_{k}=\int_{f_{k}^{* *}}^{f}\left(1-\frac{f_{k}^{* *}}{x}\right) d x, \\
f_{k}^{* *} \in W=\left\{S, I_{1}, I_{2}, I_{3}, I_{4}, A\right\},
\end{gathered}
$$

for

where $k=1,2,3,4,5,6$. This vividly shows that $B_{k}$ is positive definite, continuous and differentiable in $\Gamma$. Hence, $B_{k} \in C^{\prime}\left[\Gamma, \mathbb{R}^{+}\right]$. Differentiating $B$ partially with respect to each $f_{k}$ we have

$$
M_{1}(X, Y)=\left[\begin{array}{c}
\frac{\beta \alpha_{1}\left(\sigma_{1} I_{1}+\sigma_{2} I_{2}+I_{3}\right) S}{N}-K_{1} I_{1} \\
\frac{\beta \alpha_{2}\left(\sigma_{1} I_{1}+\sigma_{2} I_{2}+I_{3}\right) S}{N}-K_{2} I_{2} \\
\frac{\beta \alpha_{3}\left(\sigma_{1} I_{1}+\sigma_{2} I_{2}+I_{3}\right) S}{N}-K_{3} I_{3} \\
\gamma_{1} I_{2}-\mu I_{4} \\
\rho_{1} I_{1}+\rho_{2} I_{3}-K_{5} A
\end{array}\right]
$$

so that

$$
\frac{\partial B}{\partial f_{k}}=0 \Longrightarrow A_{k}\left(1-\frac{f_{k}^{* *}}{f_{k}}\right)=0 .
$$


R. Musa, R. Willie, N. Parumasur, Analysis of a virus-resistant HIV-1 model with behavior change in ...

Differentiating (32) again partially with respect to each $f_{k}$ gives

$$
\frac{\partial^{2} B}{\partial f_{k}^{2}}=\frac{A_{k} f_{k}^{* *}}{f_{k}^{2}}, k=1, \ldots, 6 .
$$

From [32, if $f_{k}=f_{k}^{* *}$, then $S=S^{* *}, I_{1}=$ $I_{1}^{* *}, I_{2}=I_{2}^{* *}, I_{3}=I_{3}^{* *}, I_{4}=I_{4}^{* *}, A=A^{* *}$. This clearly shows that the endemic equilibrium point is the only stationary point of $B$. Since 30 is always positive, it means that the endemic equilibrium is a global minimum point of the function $B$ for all $f_{k} \in \Gamma \subseteq \mathbb{R}_{+}^{6}$. Next is to establish that the function $B$ is a Lyapunov function which can be done by proving that $B$ is negative definite. The time derivative of $B$ is given by

$$
\frac{d B}{d t}=\sum_{k=1}^{6} A_{k}\left(1-\frac{f_{k}^{* *}}{f_{k}}\right) \dot{f}_{k}
$$

which is negative definite for all time $t>0$. It is worth noting here that for all $f_{k}^{* *} \in \Gamma, \dot{f}_{k} \leq \dot{N}$ which makes equation (34) to be

$$
\frac{d B}{d t} \leq \sum_{k=1}^{6} A_{k}\left(1-\frac{f_{k}^{* *}}{f_{k}}\right) \dot{N} .
$$

From equation 12 , we obtain the derivative

$$
\frac{d N}{d t}=\mu\left(\frac{B}{\mu}-N(0)\right) \exp (-\mu t) .
$$

Substituting (36) in (35), we have

$$
\frac{d B}{d t} \leq \sum_{k=1}^{6} A_{k}\left(1-\frac{f_{k}^{* *}}{f_{k}}\right) \mu\left(\frac{B}{\mu}-N(0)\right) \exp (-\mu t) .
$$

When $t \rightarrow \infty, \frac{d B}{d t} \leq 0$ which means that the total initial population $N(0)$ is within the basin $\Gamma$ i.e. $N(0) \leq \frac{B}{\mu}$. Also when the initial population is outside the basin of attraction i.e. $N(0) \geq \frac{B}{\mu}$ as $t \rightarrow \infty$, then $\frac{d B}{d t} \leq 0$ and hence, the righthand side of (37) is negative definite. This proves that irrespective of the size of the initial population $N(0)$, the left hand side is always less or equal to zero as $t>0$. This consequently clarifies that the constructed function $B$ is a Lyapunov type and can be used to establish the global stability of the system. Moreover, $\frac{d B}{d t}=0$ if and only if

$$
\begin{aligned}
& S=S^{* *}, I_{1}=I_{1}^{* *}, I_{1}=I_{1}^{* *}, I_{2}=I_{2}^{* *}, \\
& I_{3}=I_{3}^{* *}, I_{4}=I_{4}^{* *}, A=A^{* *},
\end{aligned}
$$

and the largest positive invariant subset of $\Gamma$ that satisfies $\frac{d B}{d t}=0$ is the singleton $\psi_{1}^{* *}$. Hence, $\psi_{1}^{* *}$ is a unique endemic equilibrium point of the system (13)-(18) which is GAS in $\Gamma$.

\section{F. Analysis of the Full Model}

\section{G. Local Stability of DFE}

In this section, we shall analyze the full model just as we did for the sub-model in the previous section. It is worth noting that the full model has the same DFE as the sub-model given by equation (20) which exists in the same region $\Gamma$. We employ the same next generation matrix to establish the reproduction number as follows:

$$
\begin{aligned}
\mathcal{F} & =\left[\begin{array}{ccccc}
\alpha_{1} \sigma_{1} \beta & \beta \sigma_{2} \alpha_{1} & \beta \alpha_{1} & \beta \alpha_{1} \sigma_{3} & 0 \\
\alpha_{2} \sigma_{1} \beta & \beta \sigma_{2} \alpha_{2} & \beta \alpha_{2} & \beta \alpha_{2} \sigma_{3} & 0 \\
\alpha_{3} \sigma_{1} \beta & \beta \sigma_{2} \alpha_{3} & \beta \alpha_{3} & \beta \alpha_{3} \sigma_{3} & 0 \\
0 & 0 & 0 & 0 & 0 \\
0 & 0 & 0 & 0 & 0
\end{array}\right] \\
\mathcal{V} & =\left[\begin{array}{ccccc}
K_{1} & 0 & 0 & 0 & 0 \\
0 & K_{2} & 0 & -\gamma_{2} & 0 \\
0 & 0 & K_{3} & 0 & 0 \\
0 & -\gamma_{1} & 0 & K_{4} & 0 \\
-\rho_{1} & 0 & -\rho_{2} & 0 & K_{5}
\end{array}\right]
\end{aligned}
$$

Taking $\rho$ as the spectral radius (magnitude of the dominate eigenvalue) of the next generation matrix $\mathcal{F} \mathcal{V}^{-1}$, the reproduction number is given by

$$
\mathcal{R}_{T}=\left[\frac{P+Q}{K_{1} K_{3}\left(K_{2} K_{4}-\gamma_{1} \gamma_{2}\right)}\right],
$$

where

$$
\begin{aligned}
& P=\left(K_{2} K_{4}-\gamma_{1} \gamma_{2}\right)\left(\alpha_{1} K_{3} \sigma_{1}+\alpha_{3} K_{1}\right), \\
& Q=\alpha_{2} K_{1} K_{3}\left(\gamma_{1} \sigma_{3}+K_{4} \sigma_{2}\right) .
\end{aligned}
$$

Lemma IV.4. The disease-free equilibrium point (DFE) of the full model (4)-(9) with partial abstinence is locally asymptotically stable (LAS) if $\mathcal{R}_{T}<1$ and unstable otherwise. 
R. Musa, R. Willie, N. Parumasur, Analysis of a virus-resistant HIV-1 model with behavior change in ...

\section{H. Existence of Endemic Equilibrium}

The full model with partial abstinence has a unique positive endemic equilibrium point (EEP). This is the point where at least one of the virus infected compartments is non-zero. Let

$$
\psi^{* *}=\left(S^{* *}, I_{1}^{* *}, I_{2}^{* *}, I_{3}^{* *}, I_{4}^{* *}, A^{* *}\right)
$$

be the endemic equilibrium point. We further define the force of infection as

$$
\lambda^{* *}=\frac{\beta\left(I_{3}^{* *}+\sigma_{1} I_{1}^{* *}+\sigma_{2} I_{2}^{* *}+\sigma_{3} I_{4}^{* *}\right)}{N^{* *}} .
$$

Solving equation (4)-(9) in terms of the force of infection $\lambda^{* *}$ at steady-state we obtain:

$$
\begin{aligned}
S^{* *} & =\frac{B}{\mu+\left(\alpha_{1}+\alpha_{2}+\alpha_{3}\right) \lambda^{* *}}, \\
I_{1}^{* *} & =\frac{\alpha_{1} B \lambda^{* *}}{K_{1}\left[\mu+\left(\alpha_{1}+\alpha_{2}+\alpha_{3}\right) \lambda^{* *}\right]}, \\
I_{3}^{* *} & =\frac{B \lambda^{* *}}{K_{3}\left[\mu+\left(\alpha_{1}+\alpha_{2}+\alpha_{3}\right) \lambda^{* *}\right]}, \\
A^{* *} & =\frac{B \lambda^{* *}\left(\rho_{1} \alpha_{1} K_{3}+K_{1} \rho_{2}\right)}{K_{1} K_{3} K_{5}\left[\mu+\left(\alpha_{1}+\alpha_{2}+\alpha_{3}\right) \lambda^{* *}\right]}, \\
I_{2}^{* *} & =\frac{\alpha_{2} B \lambda^{* *} K_{4}}{f_{1}\left(K_{2} K_{4}-\gamma_{1} \gamma_{2}\right)}, \\
N^{* *} & =\frac{B K_{1} K_{3} K_{5} f_{1}-\tau B \lambda^{* *}\left(\rho_{1} \alpha_{1} K_{3}+K_{1} \rho_{2}\right)}{\mu K_{1} K_{3} K_{5} f_{1}}, \\
I_{4}^{* *} & =\frac{\gamma_{1} B \alpha_{2} \lambda^{* *} K_{4}}{K_{4}\left[\mu+\left(\alpha_{1}+\alpha_{2}+\alpha_{3}\right) \lambda^{* *}\right]\left(K_{2} K_{4}-\gamma_{1} \gamma_{2}\right)},
\end{aligned}
$$

where $f_{1}=\mu+\left(\alpha_{1}+\alpha_{2}+\alpha_{3}\right) \lambda^{* *}$. Substituting all the equations in 41) into 40, it can be shown that the non-zero equilibria of the model satisfy the following linear equation in terms of $\lambda^{* *}$ :

$$
a_{2} \lambda^{* *}+a_{3}=0
$$

where

$$
\begin{aligned}
a_{2}= & \alpha_{3} \mu K_{1}\left(\rho_{2}+\mu+\tau\right)+\alpha_{1} K_{3} \mu\left(\mu+\rho_{1}+\tau\right) \\
& +K_{1} K_{3} K_{5} \alpha_{2}>0 \\
a_{3}= & \mu K_{1} K_{3} K_{5}\left(1-\mathcal{R}_{T}\right) .
\end{aligned}
$$

Clearly, $a_{2}>0, a_{3} \geq 0$ if and only if $\mathcal{R}_{T} \leq 1$ so that $\lambda^{* *}=-\frac{a_{3}}{a_{2}} \leq 0$ which shows no existence of positive endemic equilibrium whenever $\mathcal{R}_{T} \leq 1$.
Hence, the endemic equilibrium point $\psi^{* *}$ exists and unique whenever $\mathcal{R}_{T}>1$. We claim the following result.

Lemma IV.5. The endemic equilibrium point (EEP) of the full model (4)-(9) with partial abstinence is locally asymptotically stable (LAS) if $\mathcal{R}_{T}>1$.

\section{Global Stability of DFE of the full model}

We will establish the proof using the same approach as in Section IV.C as follows:

$$
\begin{aligned}
& \hat{M}(X, Y)=J Y-M(X, Y) \\
& =\left[\begin{array}{c}
\frac{\beta \alpha_{1}\left(\sigma_{1} I_{1}+\sigma_{2} I_{2}+I_{3}+\sigma_{3} I_{4}\right) S^{*}}{N^{*}}-K_{1} I_{1} \\
\frac{\beta \alpha_{2}\left(\sigma_{1} I_{1}+\sigma_{2} I_{2}+I_{3}+\sigma_{3} I_{4}\right) S^{*}}{N^{*}}-K_{2} I_{2}+\gamma_{2} I_{4} \\
\frac{\beta\left(\sigma_{1} I_{1}+\sigma_{2} I_{2}+I_{3}+\sigma_{3} I_{4}\right) S^{*}}{N^{*}}-K_{3} I_{3} \\
\gamma_{1} I_{2}-K_{4} I_{4}+\rho_{2} I_{3}-K_{5} A
\end{array}\right] \\
& -\left[\begin{array}{c}
\frac{\beta \alpha_{1}\left(\sigma_{1} I_{1}+\sigma_{2} I_{2}+I_{3}+\sigma_{3} I_{4}\right) S^{*}}{N^{*}}-K_{1} I_{1} \\
\frac{\beta \alpha_{2}\left(\sigma_{1} I_{1}+\sigma_{2} I_{2}+I_{3}+\sigma_{3} I_{4}\right) S^{*}}{N^{*}}-K_{2} I_{2}+\gamma_{2} I_{4} \\
\frac{\beta\left(\sigma_{1} I_{1}+\sigma_{2} I_{2}+I_{3}+\sigma_{3} I_{4}\right) S^{*}}{N^{*}}-K_{3} I_{3} \\
\gamma_{1} I_{2}-K_{4} I_{4} \\
\rho_{1} I_{1}+\rho_{2} I_{3}-K_{5} A \\
0 \\
0 \\
\beta \alpha_{1}\left(\sigma_{1} I_{1}+\sigma_{2} I_{2}+I_{3}+\sigma_{3} I_{4}\right)\left(1-\frac{S}{N}\right) \\
\beta \alpha_{2}\left(\sigma_{1} I_{1}+\sigma_{2} I_{2}+I_{3}+\sigma_{3} I_{4}\right)\left(1-\frac{S}{N}\right) \\
\beta \alpha_{3}\left(\sigma_{1} I_{1}+\sigma_{2} I_{2}+I_{3}+\sigma_{3} I_{4}\right)\left(1-\frac{S}{N}\right) \\
0 \\
0
\end{array}\right) \geq 0,
\end{aligned}
$$

where $\frac{S^{*}}{N^{*}} \leq 1$ at DFE and since $S \leq N$, this shows that $\hat{M}(X, Y) \geq 0$. It can be seen that $\lim _{t \rightarrow \infty} X(t)=X^{o}$ and $J$ is an M-matrix, thus $X^{o}$ is globally asymptotically stable, hence, $\mathbf{N}_{1}$ is satisfied. Also, $\hat{M}(X, Y) \geq 0$ for $(X, Y) \in \Omega_{m}$. Hence, $\mathbf{N}_{2}$ is satisfied and $E^{o}$ is globally asymptotically stable whenever $\mathcal{R}_{T}<1$. 
R. Musa, R. Willie, N. Parumasur, Analysis of a virus-resistant HIV-1 model with behavior change in ...

\section{J. Global Stability of The Endemic Equilibrium}

We consider the special case where the virusinduced death rate $\tau$ is negligible. This is very much realistic since HIV-1 positive individuals under treatment can live many years hail and healthy without dying of the virus. Substituting $\tau=0$ into (11), as $t \rightarrow \infty$ gives $N \rightarrow \frac{B}{\mu}$. Putting this in equation (1), we have

$$
\lambda=\beta_{1}\left(I_{3}+\sigma_{1} I_{1}+\sigma_{2} I_{2}+\sigma_{3} I_{4}\right)
$$

where $\beta_{1}=\frac{\beta \mu}{B}$.

Theorem IV.6. The endemic equilibrium point of the full model (4)-(9) is globally asymptotically stable $(G A S)$ whenever $\mathcal{R}_{T}>1$.

Proof: Since Lemma IV.5 has already been established, we construct the following non-linear Lyapunov function for the system (4)-(9) as follows:

$$
\begin{aligned}
L & =\frac{\beta_{1}}{f_{2} \mu} \int_{S^{*}}^{S}\left(1-\frac{S^{*}}{x}\right) d x+\frac{1}{\alpha_{1} \alpha_{2}} \int_{I_{1}^{*}}^{I_{1}}\left(1-\frac{I_{1}^{*}}{x}\right) d x \\
& +\frac{\gamma_{2}}{\alpha_{2}} \int_{I_{2}^{*}}^{I_{2}}\left(1-\frac{I_{2}^{*}}{x}\right) d x+\frac{\alpha_{1}}{\beta_{1} \alpha_{3}} \int_{I_{3}^{*}}^{I_{3}}\left(1-\frac{I_{3}^{*}}{x}\right) d x \\
& +\frac{\beta_{1}^{2}}{K_{2} K_{3} \gamma_{1}} \int_{I_{4}^{*}}^{I_{4}}\left(1-\frac{I_{4}^{*}}{x}\right) d x+\frac{1}{\rho_{1} \rho_{2}} \int_{A^{*}}^{A}\left(1-\frac{A^{*}}{x}\right) d x .
\end{aligned}
$$

The derivative of $L$ along the solution of the system (4)-9) is given by

$$
\begin{aligned}
\dot{L} & =\frac{\beta_{1}}{f_{2} \mu}\left(1-\frac{S^{*}}{S}\right) \dot{S}+\frac{1}{\alpha_{1} \alpha_{2}}\left(1-\frac{I_{1}^{*}}{I_{1}}\right) \dot{I}_{1} \\
& +\frac{\gamma_{2}}{\alpha_{2}}\left(1-\frac{I_{2}^{*}}{I_{2}}\right) \dot{I}_{2}+\frac{\alpha_{1}}{\beta_{1} \alpha_{3}}\left(1-\frac{I_{3}^{*}}{I_{3}}\right) \dot{I}_{3} \\
& +\frac{\beta_{1}^{2}}{K_{2} K_{3} \gamma_{1}}\left(1-\frac{I_{4}^{*}}{I_{4}}\right) \dot{I}_{4}+\frac{1}{\rho_{1} \rho_{2}}\left(1-\frac{A^{*}}{A}\right) \dot{A} .
\end{aligned}
$$

Using (4)-(9), we have

$$
\dot{L}=\frac{\beta_{1}}{f_{2} \mu}\left[B-\left(f_{2} \lambda+\mu\right) S-\frac{S^{* *}}{S}\left\{B-\left(f_{2} \lambda+\mu\right) S\right\}\right]
$$

$$
\begin{gathered}
+\frac{1}{\alpha_{1} \alpha_{2}}\left[\alpha_{1} \lambda S-K_{1} I_{1}-\frac{I_{1}^{* *}}{I_{1}}\left\{\alpha_{1} \lambda S-K_{1} I_{1}\right\}\right]+ \\
\frac{\gamma_{2}}{\alpha_{2}}\left[\alpha_{2} \lambda S+\gamma_{2} I_{4}-K_{2} I_{2}-\frac{I_{2}^{* *}}{I_{2}}\left\{\alpha_{2} \lambda S+\gamma_{2} I_{4}-K_{2} I_{2}\right\}\right] \\
+\frac{\alpha_{1}}{\beta_{1} \alpha_{3}}\left[\alpha_{3} \lambda S-K_{3} I_{3}-\frac{I_{3}^{* *}}{I_{3}}\left\{\alpha_{3} \lambda S-K_{3} I_{3}\right\}\right] \\
+\frac{\beta_{1}^{2}}{K_{2} K_{3} \gamma_{1}}\left[\gamma_{1} I_{2}-K_{4} I_{4}-\frac{I_{4}^{* *}}{I_{4}}\left\{\gamma_{1} I_{2}-K_{4} I_{4}\right\}\right] \\
+\frac{1}{\rho_{1} \rho_{2}}\left[\rho_{1} I_{1}+\rho_{2} I_{3}-K_{5} A\right. \\
\left.\quad-\frac{A^{* *}}{A}\left\{\rho_{1} I_{1}+\rho_{2} I_{3}-K_{5} A\right\}\right],
\end{gathered}
$$

where $f_{2}=\alpha_{1}+\alpha_{2}+\alpha_{3}$. At endemic equilibrium point of (4)-(9), we have the following expressions.

$$
\begin{aligned}
& B=\mu S^{* *}+f_{2}\left(I_{3}^{* *}+\sigma_{1} I_{1}^{* *}+\sigma_{2} I_{2}^{* *}+\sigma_{3} I_{4}^{* *}\right) S^{* *} \\
& K_{1}=\frac{\alpha_{1} \beta_{1}\left(I_{3}^{* *}+\sigma_{1} I_{1}^{* *}+\sigma_{2} I_{2}^{* *}+\sigma_{3} I_{4}^{* *}\right) S^{* *}}{I_{1}^{* *}} \\
& K_{2}=\frac{\gamma_{2} I_{4}^{* *}+\alpha_{2} \beta_{1}\left(I_{3}^{* *}+\sigma_{1} I_{1}^{* *}+\sigma_{2} I_{2}^{* *}+\sigma_{3} I_{4}^{* *}\right) S^{* *}}{I_{2}^{* *}} \\
& K_{3}=\frac{\alpha_{3} \beta_{1}\left(I_{3}^{* *}+\sigma_{1} I_{1}^{* *}+\sigma_{2} I_{2}^{* *}+\sigma_{3} I_{4}^{* *}\right) S^{* *}}{I_{3}^{* *}} \\
& K_{4}=\frac{\gamma_{1} I_{2}^{* *}}{I_{4}^{* *}}, \\
& K_{5}=\frac{\rho_{1} I_{1}^{* *}+\rho_{2} I_{3}^{* *}}{A^{* *}}
\end{aligned}
$$

Substituting expressions in (47) into (46), after some simplifications and factorization, we have

$$
\begin{aligned}
\dot{L} & =\frac{\beta_{1} S^{* *}}{f_{2}}\left(2-\frac{S}{S^{* *}}-\frac{S^{* *}}{S}\right) \\
+ & \frac{\beta_{1}^{2}}{\mu}\left(I_{3}^{* *}+\sigma_{1} I_{1}^{* *}+\sigma_{2} I_{2}^{* *}+\sigma_{3} I_{4}^{* *}\right) S^{* *}\left(2-\frac{S}{S^{* *}}-\frac{S^{* *}}{S}\right) \\
+ & \frac{\beta_{1}}{\alpha_{2}}\left[\left(I_{3}^{* *}+\sigma_{1} I_{1}^{* *}+\sigma_{2} I_{2}^{* *}+\sigma_{3} I_{4}^{* *}\right) S^{* *}\left(2-\frac{I_{1}}{I_{1}^{* *}}-\frac{I_{1}^{* *}}{I_{1}}\right)\right. \\
& \left.+\left(I_{3}+\sigma_{1} I_{1}+\sigma_{2} I_{2}+\sigma_{3} I_{4}\right) S\left(2-\frac{I_{1}}{I_{1}^{* *}}-\frac{I_{1}^{* *}}{I_{1}}\right)\right] \\
& +\left(\frac{1}{\rho_{2}}+\frac{1}{\rho_{1}}\right)\left(2-\frac{A}{A^{* *}}-\frac{A^{* *}}{A}\right)
\end{aligned}
$$


R. Musa, R. Willie, N. Parumasur, Analysis of a virus-resistant HIV-1 model with behavior change in ...

$+\gamma_{2}\left[\beta_{1}\left(I_{3}+\sigma_{1} I_{1}+\sigma_{2} I_{2}+\sigma_{3} I_{4}\right) S\left(2-\frac{I_{2}}{I_{2}^{* *}}-\frac{I_{2}^{* *}}{I_{2}}\right)\right.$

$\left.+\beta_{1}\left(I_{3}^{* *}+\sigma_{1} I_{1}^{* *}+\sigma_{2} I_{2}^{* *}+\sigma_{3} I_{4}^{* *}\right) S^{* *}\left(2-\frac{I_{2}}{I_{2}^{* *}}-\frac{I_{2}^{* *}}{I_{2}}\right)\right]$

$+\left(\frac{\gamma_{2}^{2}}{\alpha_{2}}+\frac{\alpha_{1} \beta_{1}^{2}}{K_{2} K_{3}}\right) \frac{I_{2}^{2} I_{3}^{* *}}{I_{4}^{* *}}\left(3-\frac{I_{2}^{* *}}{I_{2}}-\frac{I_{2} I_{3}^{* *}}{I_{2}^{* *} I_{3}}-\frac{I_{2}^{* *} I_{3} I_{4}}{I_{2} I_{3}^{* *} I_{4}^{* *}}\right)$.

Consequently, since the arithmetic mean exceeds the geometric mean, then we have

$$
\begin{aligned}
& 2-\frac{S}{S^{* *}}-\frac{S^{* *}}{S} \leq 0, \\
& 2-\frac{A}{A^{* *}}-\frac{A^{* *}}{A} \leq 0, \\
& 2-\frac{I_{1}}{I_{1}^{* *}}-\frac{I_{1}^{* *}}{I_{1}} \leq 0, \\
& 2-\frac{I_{2}}{I_{2}^{* *}}-\frac{I_{2}^{* *}}{I_{2}} \leq 0, \\
& 3-\frac{I_{2}^{* *}}{I_{2}}-\frac{I_{2} I_{3}^{* *}}{I_{2}^{* *} I_{3}}-\frac{I_{2}^{* *} I_{3} I_{4}}{I_{2} I_{3}^{* *} I_{4}^{* *}} \leq 0 .
\end{aligned}
$$

Since $S \geq 0, I_{1} \geq 0, I_{2} \geq 0, I_{3} \geq 0, I_{4} \geq$ $0, A \geq 0$ and Lemma IV.5 is satisfied, it follows that $\dot{\dot{L}} \leq 0$ since all other model parameters are non-negative for $\mathcal{R}_{T}>1$. Furthermore, $\dot{L}=0$ if and only if $S=S^{* *}, I_{1}=I_{1}^{* *}, I_{2}=I_{2}^{* *}, I_{3}=$ $I_{3}^{* *}, I_{4}=I_{4}^{* *}, A=A^{* *}$. Thus, $L$ is a Lyapunov function of the subsystem (4)-(9) on $\Gamma$. It therefore follows by LaSalle's Invariance Principle [16] that the subsystem (4)-(9) has a globally asymptotically stable endemic equilibrium point $\psi^{* *}$. The result presented here shows that for a special case $(\tau=0)$, the virus will consistently persist in the community whenever the associated reproduction number $\mathcal{R}_{T}>1$.

\section{Numerical Simulation and Discussion OF RESULTS}

In this section, we shall carry out the numerical simulation of the model to corroborate the analytic results. We shall solve the model equation (4)(9) numerically and present the results graphically using Maple 18 and Python mathematical software. A 3D surface plot shall also be presented to examine the relationship between the reproduction number, the partial abstinence rate $\gamma_{2}$ and $\sigma_{3}$ which is the modification parameter which account for the assumed reduction of infectiousness by the behavior change class $I_{4}$.

Table 1: Hypothetical Value of Parameters

\begin{tabular}{lll}
\hline Parameter & Value (per year) & Source \\
\hline$B$ & 5600 & Estimated \\
$\alpha_{1}$ & 0.25 & Estimated \\
$\alpha_{2}$ & 0.10 & Estimated \\
$\beta$ & 0.015 & Estimated \\
$\mu$ & 0.016 & Estimated \\
$\alpha_{3}$ & 0.65 & Estimated \\
$\rho_{1}$ & 0.12 & Estimated \\
$\gamma_{1}$ & 1.00 & {$[30],[20]$} \\
$\gamma_{2}$ & 0.95 & Estimated \\
$\rho_{2}$ & 0.75 & Estimated \\
$\tau$ & 0.0909 & {$[11]$} \\
$\sigma_{1}$ & 0.85 & Estimated \\
$\sigma_{2}$ & 0.55 & Estimated \\
$\sigma_{3}$ & 0.008 & {$[29]$} \\
\hline
\end{tabular}

\section{Table 2: Initial Conditions}

\begin{tabular}{llllll}
\hline$S(0)$ & $I_{1}(0)$ & $I_{2}(0)$ & $I_{3}(0)$ & $I_{4}(0)$ & $A(0)$ \\
\hline 450 & 10 & 8 & 5 & 10 & 15 \\
400 & 40 & 25 & 20 & 10 & 5 \\
300 & 70 & 50 & 40 & 30 & 10 \\
200 & 95 & 65 & 53 & 47 & 40 \\
100 & 120 & 88 & 67 & 65 & 60 \\
\hline
\end{tabular}

To start with, we will show numerically that the disease-free equilibrium $\psi^{*}$ is locally asymptotically stable. The parameter values presented in Table 1 and the initial conditions shown in Table 2 shall be used.

Considering the case when the reproduction number is less than unity i.e. $\mathcal{R}_{T}=0.025<1$, the graphical solution of model equation (4)-(9) is given in fig. 2 - fig.7. It can be seen that only the susceptible population $S=500$ survive while the infected population in the slow progression class $I_{1}$, non progression class $I_{2}$, fast progression class $I_{3}$, behavior change class $I_{4}$ and AIDS class $A$ goes into extinction. This confirms that the DFE of (4)-(9) as presented in Lemma (IV.4) is locally asymptotically stable whenever $\mathcal{R}_{T}<1$. 

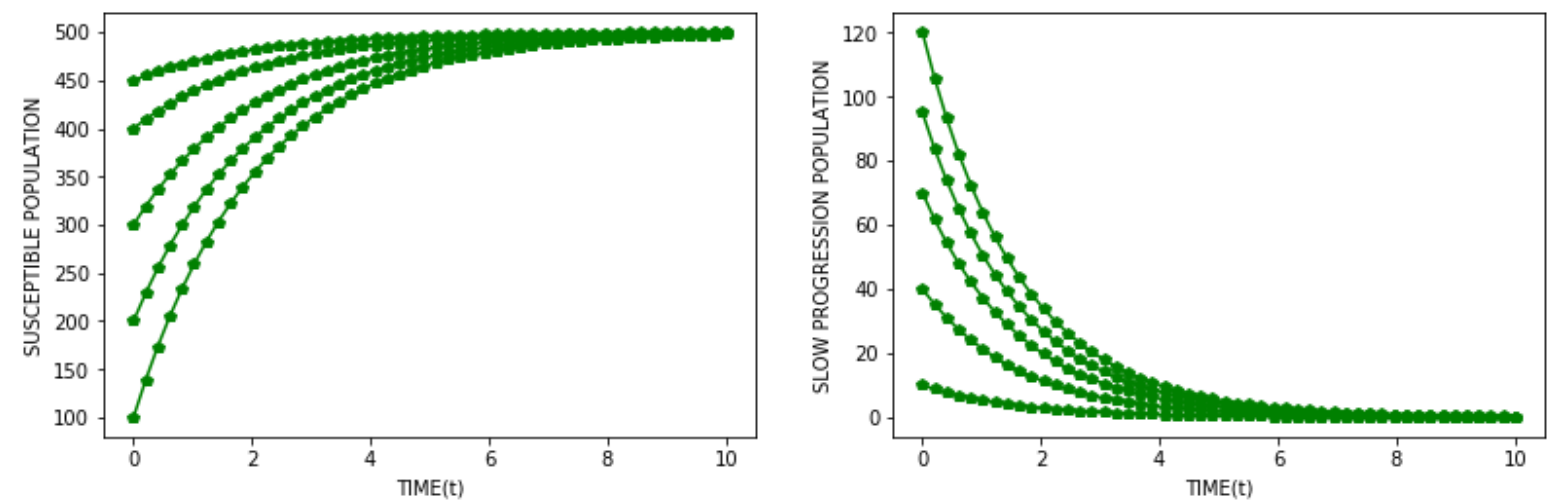

Fig. 2 and Fig. 3 Showing The Behavior of Both Susceptible and Slow Progression Populations when $\mathcal{R}_{T}$ is Less Than Unity.
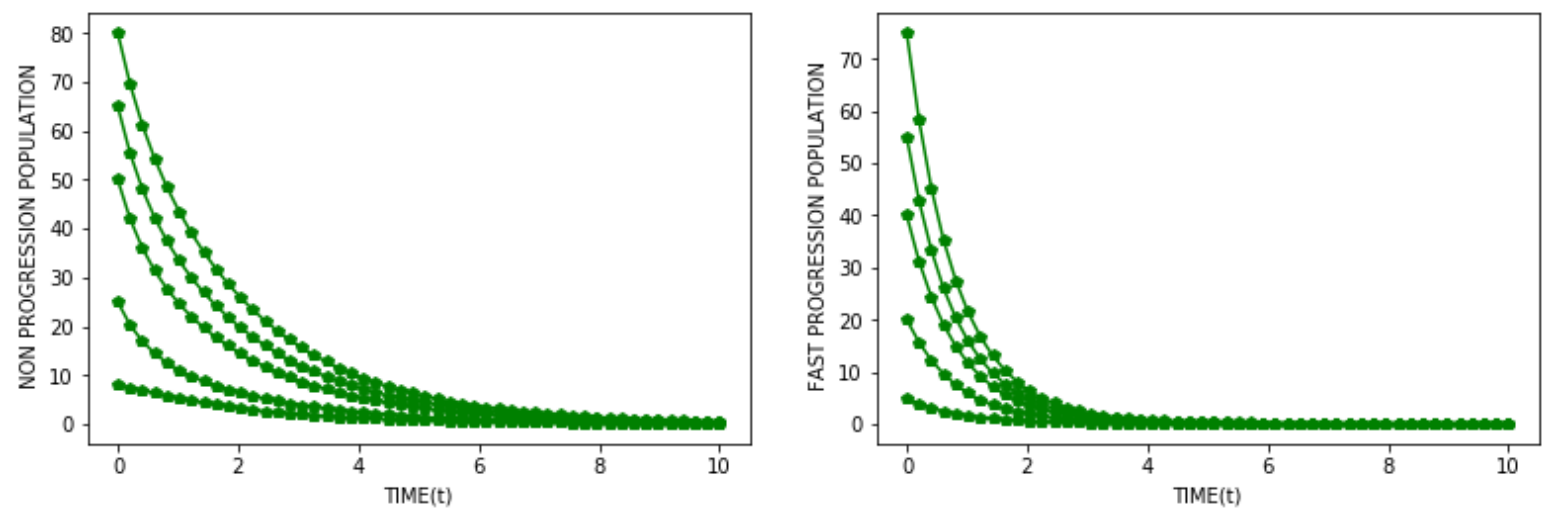

Fig. 4 and Fig. 5 Showing The Behavior of Both Non Progression and Fast Progression Populations when $\mathcal{R}_{T}$ is less than Unity.
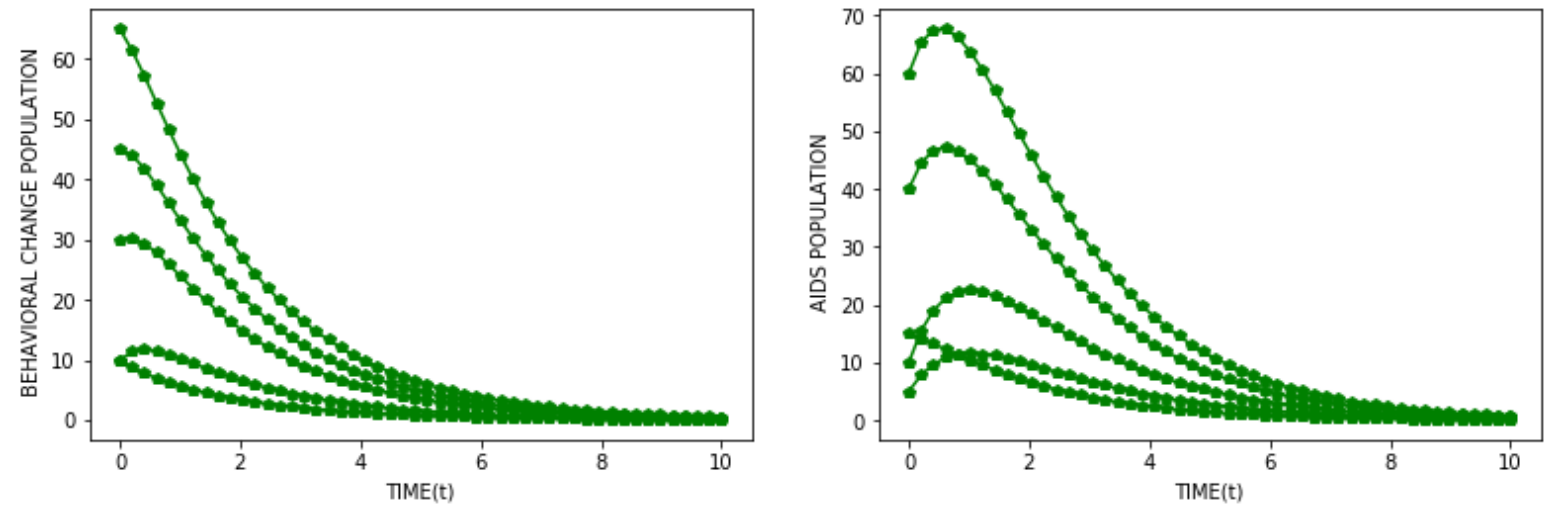

Fig. 6 and Fig. 7 Showing The Behavior of Both Fast Progression and AIDS Populations when $\mathcal{R}_{T}$ is Less Than Unity. 
R. Musa, R. Willie, N. Parumasur, Analysis of a virus-resistant HIV-1 model with behavior change in ...
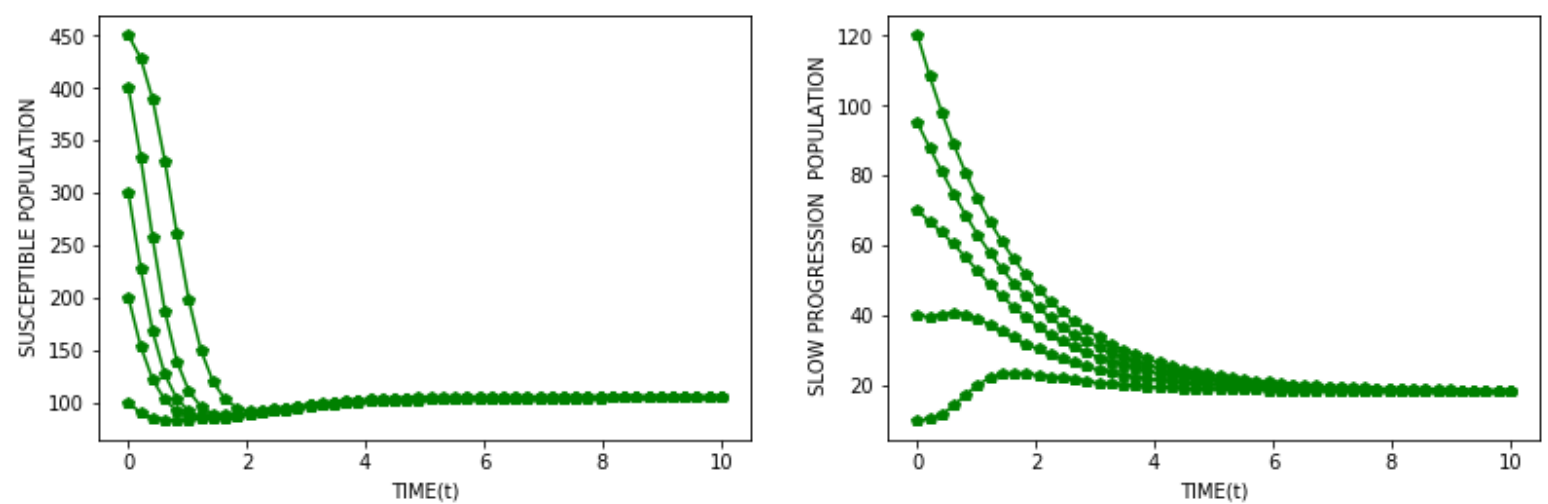

Fig. 8 and Fig. 9 Showing The Behavior of Both Susceptible and Slow Progression Populations when $\mathcal{R}_{T}$ is Greater Than Unity.
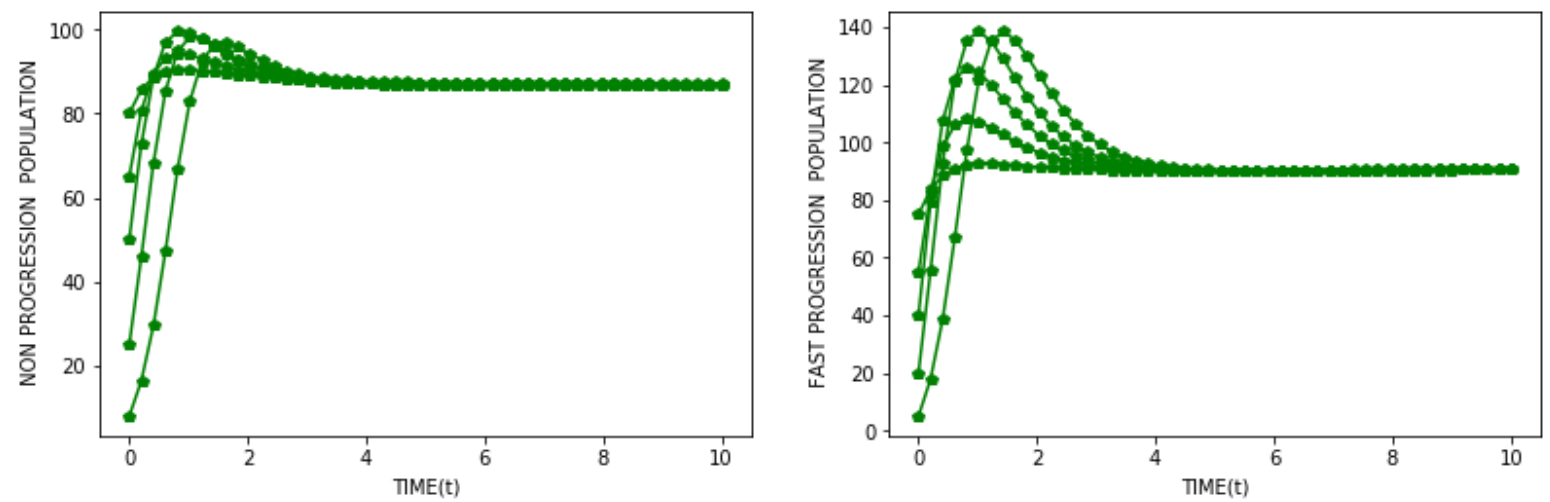

Fig. 10 and Fig. 11 Showing The Behavior of Both Non Progression and Fast Progression Populations when $\mathcal{R}_{T}$ is Greater Than Unity.
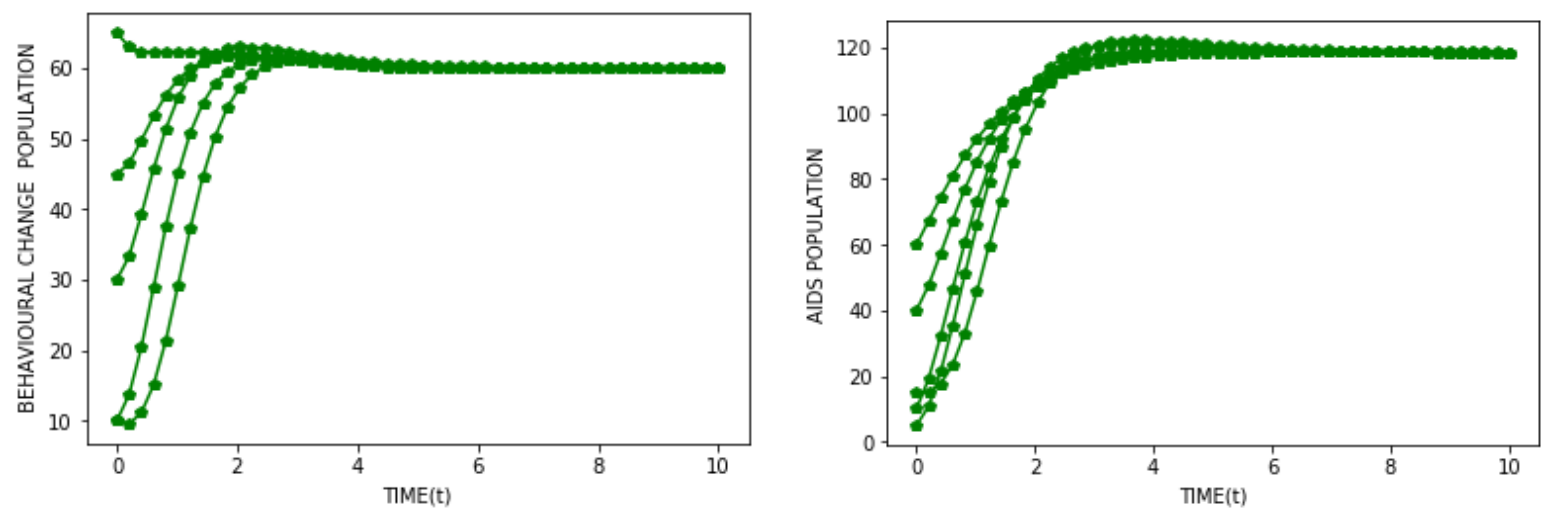

Fig. 12 and Fig. 13 Showing The Behavior of Both Fast Progression and AIDS Populations when $\mathcal{R}_{T}$ is Greater Than Unity. 
R. Musa, R. Willie, N. Parumasur, Analysis of a virus-resistant HIV-1 model with behavior change in ...

Considering the case when the reproduction number is greater than unity i.e. $\mathcal{R}_{T}=5.903>1$, the graphical solution of model equation (4)-9) is given in fig.8 - fig.13. It can be observed that when the reproduction number $\mathcal{R}_{T}>1$, all the populations survive that's

$$
\begin{aligned}
& {\left[S^{* *}, I_{1}^{* *}, I_{2}^{* *}, I_{3}^{* *}, I_{4}^{* *}, A^{* *}\right]=} \\
& {[0.0155,0.6575,0.9452,0.8105,0.5421,0.7813],}
\end{aligned}
$$

which clearly indicates that the population converges or tends to the endemic equilibrium points $\psi^{* *}$ whenever $\mathcal{R}_{T}>1$. It can also be seen that the susceptible population reduces drastically because of the reproduction number being greater than unity while all the remaining infected populations increases with time. This confirms that the endemic equilibrium points $\psi^{* *}$ is locally asymptotically stable and thus, confirms the analytic results presented in Lemma IV.5.

\section{A. Effect of Partial and Total Abstinence in HIV/AIDS Transmission}

Here, we will observe the effect of partial and total abstinence in the transmission of HIV/AIDS. We simulate the reproduction number in equation (21) and (38). For the case of partial abstinence (i.e. when $\sigma_{3}=\gamma_{2} \neq 0$ ), with the parameter values in Table 1, when $\gamma_{2}=0.95$ and $\sigma_{3}=0.008$, $\mathcal{R}_{T}=0.025$. For the case of total abstinence (i.e. when $\sigma_{3}=\gamma_{2}=0$ ), with the parameter values in Table $1, \mathcal{R}_{T}^{\prime}=0.020$ meaning that $\mathcal{R}_{T}^{\prime}$ for the total abstinence is less than $\mathcal{R}_{T}$ for the partial abstinence. Since our aim in epidemiology is to find all possible means to reduce the reproduction number of infectious disease, it means that those that changed their sexual attitude through total abstinence from HIV/AIDS and all factors that can cause its transmission are at lower or no risk of contacting HIV/AIDS. Hence, total abstinence is one of the key factors to be safe from HIV/AIDS. Figure 14 below shows a 3D surface plot to understand more about the relationship between the reproduction number and $\gamma_{2}$ and $\sigma_{3}$.

We can easily observe that the higher the value of both $\sigma_{3}$ and $\gamma_{2}$, the higher the reproduction number and the lower their values the lower the reproduction number. The lowest reproduction number 0.018 is gotten when $\sigma_{3}=\gamma_{2}=0$ i.e. (total abstinence). Hence, total abstinence is essential in the protection against HIV/AIDS transmission.

\section{Conclusion, ACKnOWledgment And Disclosure Statement}

In this study, a new virus resistant HIV-1 model with behavior change was proposed and systematically analyzed for both partial and total abstinence from HIV/AIDS. Basic analysis of the model such as positivity solution, reproduction number, invariant region, establishment of both disease-free and endemic equilibrium points for both scenarios were carried out. The local asymptotic stability of the DFE and EE for both models whenever the associated reproduction number is less than unity and greater than unity respectively were proved. A non-linear Goh-Volterra Lyapunov function is used to prove that the endemic equilibrium point is globally asymptotically stable for the case when the virus-induced death rate $\tau=0$ while the method of Castillo-Chavez is used to prove the global asymptotic stability of the disease-free equilibrium point whenever the reproduction number is less than unity. In the numerical simulation, it was established that people with total abstinence are more protected against HIV/AIDS than those with partial abstinence and also established that the reproduction number is minimal under this same condition. Since those with resistance to HIV/AIDS do not proceed to the AIDS compartment, this also highlight the importance of HIVresistance which plays an important role in the protection against HIV/AIDS.

\section{Acknowledgment}

The authors really acknowledge and appreciate the efforts of the unknown reviewers. The first author, Rabiu Musa, acknowledges funding from the NRF and DST of South Africa through grant number 48518.

\section{Disclosure Statement}

The research work forms part of the first authors 


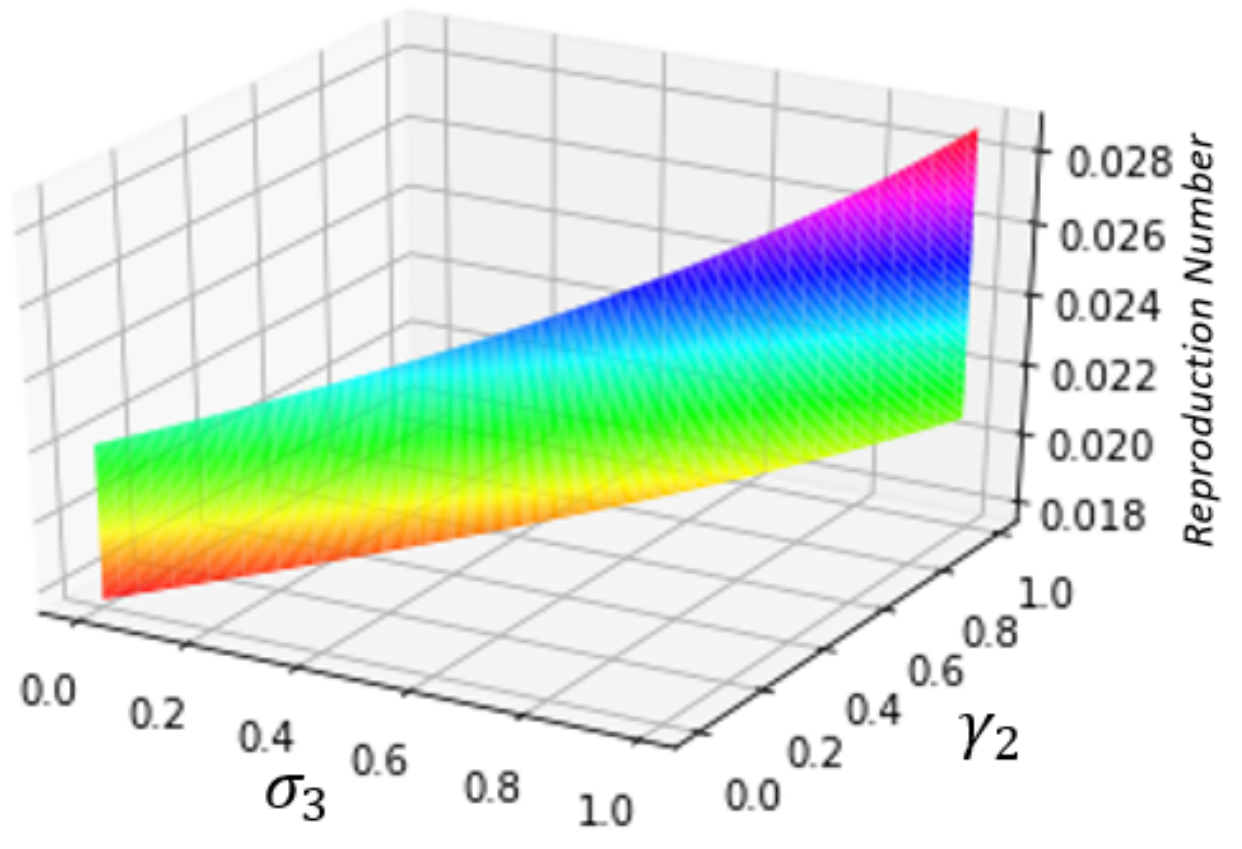

Fig. 14 Showing The Effect of $\sigma_{3}$ and $\gamma_{2}$ on the Reproduction Number Using the Parameter Values on Table 1 when $\sigma_{3}=\gamma_{2}=[0,1]$.

$\mathrm{PhD}$ work and the co-authors are his supervisors.

\section{Data Availability Statement}

The numerical data and hypothetical value of parameters used to support the findings of this research are included within the article. They are either properly referenced, assumed or estimated in Table 1.

\section{Conflict of Interest}

No conflict of interest as far as this research is concerned.

\section{REFERENCES}

[1] Afassinou, K., Chirove, F., \& Govinder, K. S. (2017). Pre-exposure prophylaxis and antiretroviral treatment interventions with drug resistance. Mathematical biosciences, 285, 92-101.

[2] Altman, Lawrence K. (3 February 2000). "A New AIDS Mystery: Prostitutes Who Have Remained Immune". NYTimes. Retrieved 2015-01-20.

[3] Blackwell, Tom (2012-02-13). "Blackwell on Health: Montreal researchers discover why some prostitutes evade HIV". National Post. Retrieved 2015-01-20. Public Health Agency of Canada have identified 15 proteins unique to those virus-free prostitutes.
[4] Biasin, M., Clerici, M., \& Piacentini, L. (2010). Innate immunity in resistance to HIV infection. The Journal of infectious diseases, 202(Supplement3), S361-S365.

[5] Castillo-Chavez, C., \& Song, B. (2004). Dynamical models of tuberculosis and their applications. Mathematical Biosciences \& Engineering, 1(2), 361.

[6] Cohen J. (2007). AIDS research. Did Merck's failed HIV vaccine cause harm? Science 318: 1048-1049.

[7] Diekmann, O., Heesterbeek, J. A. P.,\& Metz, J. A. (1990). On the definition and the computation of the basic reproduction ratio $\mathrm{R} 0$ in models for infectious diseases in heterogeneous populations. Journal of mathematical biology, 28(4), 365-382.

[8] Easterbrook PJ. Long-term non-progression in HIV infection: definitions and epidemiological issues. $J$ Infect. 1999;38(2):71-73.

[9] Elaine Mendus and Trudy Ring (2016): A genetic mutation that blocks HIV may hold the key to future treatment and, perhaps, a cure.

[10] Garcia-Calleja, J. M., Gouws, E., \& Ghys, P. D. (2006). National population based HIV prevalence surveys in sub-Saharan Africa: results and implications for HIV and AIDS estimates. Sexually transmitted infections, 82(suppl 3), iii64-iii70.

[11] Huo, H. F., Chen, R., \& Wang, X. Y. (2016). Modelling and stability of HIV/AIDS epidemic model with treatment. Applied Mathematical Modelling, 40(1314), 6550-6559. 
[12] Jesse Green (2014-06-13). "The Man Who Was Immune to AIDS". NYMag.com. Retrieved 2015-01-20.

[13] Jia, J., \& Xiao, J. (2018). Stability analysis of a disease resistance SEIRS model with nonlinear incidence rate. Advances in Difference Equations, 2018(1), 75.

[14] Julia Paoli. HIV Resistant Mutation. Available on https://www.nature.com/scitable/blog/viruses101/ HIV resistant mutation/?isForcedMobile $=Y$

[15] Khanh, N. H. (2016). Stability analysis of an influenza virus model with disease resistance. Journal of the Egyptian Mathematical Society, 24(2), 193-199.

[16] LaSalle JP. The Stability of Dynamical Systems, Regional Conference Series in Applied Mathematics. SIAM: Philadelphia, 1976.

[17] Maanvi Singh (21 September 2013). "In Life, Man Immune To HIV Helped Scientists Fight Virus". NPR.org. Retrieved 2015-01-20.

[18] Marmor, M., Hertzmark, K., Thomas, S. M., Halkitis, P. N., \& Vogler, M. (2006). Resistance to HIV infection. Journal of urban health, 83(1), 5-17.

[19] Naresh, R., Tripathi, A. and Sharma, D. (2009). Modelling and Analysis of the Spread of AIDS Epidemic with Immigration of HIV Infectives. Mathematical and Computer Modelling , 49, 880-892. https://doi.org/10. 1016/j.mcm.2008.09.013

[20] Okosun, K. O., Makinde, O. D., \& Takaidza, I. (2013). Impact of optimal control on the treatment of HIV/AIDS and screening of unaware infectives. Applied Mathematical Modelling, 37(6), 3802-3820.

[21] Padian NS, van der Straten A, Ramjee G, Chipato T, de Bruyn G, et al. (2007). Diaphragm and lubricant gel for prevention of HIV acquisition in southern African women: a randomised controlled trial. Lancet 370: 251-261.

[22] Paxton, W. A., Martin, S. R., Tse, D., O’Brien, T. R., Skurnick, J., VanDevanter, N. L., ... \& Koup, R. A. (1996). Relative resistance to HIV-1 infection of CD4 lymphocytes from persons who remain uninfected despite multiple high-risk sexual exposures. Nature medicine, 2(4), 412.

[23] Perelson, A. S., \& Nelson, P. W. (1999). Mathematical analysis of HIV-1 dynamics in vivo. SIAM review, 41(1), 3-44.
[24] Ramjee G, Govinden R, Morar NS, Mbewu A (2007). South Africa's experience of the closure of the cellulose sulphate microbicide trial. PLoS Med 4: e235.

[25] Rabiu, M., Willie, R., \& Parumasur, N. (2020). Mathematical analysis of a disease-resistant model with imperfect vaccine, quarantine and treatment. Ricerche di Matematica, 1-25.

[26] Rabiu M., Ibrahim M.O., Akinyemi S.T. (2016) : Mathematical Modeling of the Effect of Therapeutic Vaccine in the Control of Dengue Fever. Journal of the Nigerian Association of Mathematical Physics. Vol 33, PP 377-386.

[27] Rabiu M., and Akinyemi S.T. (2016): Global Analysis of Dengue Fever in a Variable Population. Journal of the Nigerian Association of Mathematical Physics. Vol 33, PP 363-376.

[28] Samson, M., Libert, F., Doranz, B. J., Rucker, J., Liesnard, C., Farber, C. M., ... \& Muyldermans, G. (1996). Resistance to HIV-1 infection in caucasian individuals bearing mutant alleles of the CCR-5 chemokine receptor gene. Nature, 382(6593), 722.

[29] Sharomi, O., \& Gumel, A. B. (2008). Dynamical analysis of a multi-strain model of HIV in the presence of anti-retroviral drugs. Journal of Biological Dynamics, 2(3), 323-345.

[30] Silva, C. J., \& Torres, D. F. (2017). Global stability for a HIV/AIDS model. arXiv preprint arXiv:1704.05806.

[31] Tripathi, A., Naresh, R., Tchuenche, J.M. and Sharma, D. (2013). Modeling the Spread of HIV-AIDS with Infective Immigrants and Time Delay. International Journal of Nonlinear Science , 16, 313-322.

[32] UNAIDS. AIDS epidemic update. Technical report, 2009. Www.unaids.org/en/KnowledgeCentre/HIV Data/EpiUpdate/EpiUpdArcHIVe/2009/default.asp

[33] University of Minnesota (2014). "Why some people may be immune to HIV-1: Clues." ScienceDaily. ScienceDaily, 20 November 2014. www.sciencedaily.com/ releases/2014/11/141120141750.htm

[34] Van den Driessche, P., \& Watmough, J. (2002). Reproduction numbers and sub-threshold endemic equilibria for compartmental models of disease transmission. Mathematical biosciences, 180(1-2), 29-48. 